\title{
Entdeckung zweier Veränderlicher.
}

\section{Var. 12.1904 Geminorum.}

Auf einer Aufnahme 1904 März $209^{\mathrm{h}} 13^{\mathrm{m}} \cdot 7$ M. Z. Kgst. stand der Veränderliche in $6^{\mathrm{h}} 3^{8^{\mathrm{m}}} \mathrm{1}+18^{\circ} 47^{\prime}(1855)$, Größe 10\% $\frac{\mathrm{m}}{\circ}$. Frühere Aufnahmen derselben Gegend ergeben:

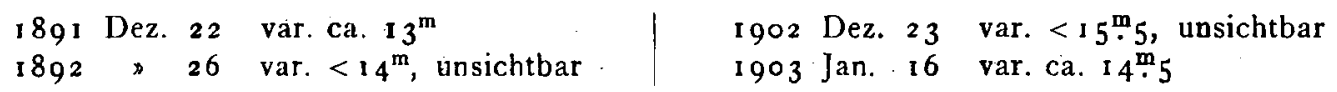

Der Stern bildet mit den beiden BD.-Sternen +1 $8: 1340$ und +1 $8^{\circ} 1343$ ein leicht kenntliches Dreieck.

\section{Var. 13.1904 Leonis.}

Auf den Platten vom 21 . Februar $190 \mathrm{r}$ stand in

$$
(1900.0) \text { I } 0^{\text {h }} 11^{m} 50^{s} .0+12^{\circ} 53^{\prime} 12^{\prime \prime}
$$

ein Stern 12. Größe, der auf den Platten vom I3. März r go s spurlos verschwunden war. Auf den älteren Aufnahmen, ebenso wie auf diesjährigen Aufnahmen ist nicht die geringste Spur des Sternes angedeutet.

Gr. Astrophys. Observatorium Königstuhl-Heidelberg, I904 März 22.

Max Wolf.

Beobachtungen von Planeten auf der k. k. Sternwarte in Wien.

\begin{tabular}{|c|c|c|c|c|c|c|c|c|c|c|c|c|}
\hline Planet & 190 & & M.Z. Wien & Gr. & & $\alpha$ ap & & $\log p \cdot \Delta$ & & app & & $\log p \cdot \Delta$ \\
\hline I $904 \mathrm{NN}$ & März & 24. & $12^{\mathrm{h}} \times 9^{\mathrm{m}} 36^{\mathrm{s}}$ & 12.8 & I $0^{h}$ & $8^{m}$ & 0.18 & 9.355 & +8 & $3^{\prime}$ & $20: 9$ & 0.764 \\
\hline $1904 \mathrm{NO}$ & 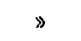 & » & II $5^{6} \cdot 3^{I}$ & 14.0 & 10 & I 7 & 7.78 & 9.259 & +13 & 20 & 56.9 & 0.712 \\
\hline (255) Oppavia & 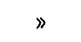 & » & $15 \quad 2936$ & 12.5 & 12 & 24 & 40.10 & 9.466 & +0 & $\mathbf{I}$ & 38.7 & $0.8 \times 5$ \\
\hline $1.904 \mathrm{NQ}$ & » & 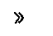 & $12 \quad 56 \quad 25$ & 13.0 & 12 & 42 & 8.20 & $8.5^{8 I}$ & - & 46 & 19.7 & 0.827 \\
\hline $1904 \mathrm{NS}$ & 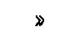 & 》 & 54 & I 2.5 & 12 & $5 t$ & 2.02 & 9.333 & + & I 2 & 54.0 & $0.76 \mathrm{I}$ \\
\hline $1904 \mathrm{NT}$ & » & $\gg$ & 143410 & 13.5 & 12 & 57 & 47.99 & 9.242 & + & 25 & 32.9 & 0.755 \\
\hline
\end{tabular}

Wien, k. k. Sternwarte, 1904 März 26.

F. Palisa.

\section{Nachträgliche Auffindung eines neuen Planeten 1902 NU.}

Nachträglich habe ich folgenden neuen Planeten 13. Größe aus dem Jahre I902 gefunden:

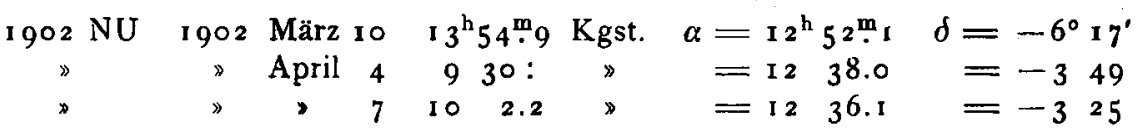

Gr. Astrophys. Observatorium Königstuhl-Heidelberg, I 904 März 17.

M. Wolf.

\section{Aufforderung betr. Fehler in Sternkatalogen.}

Hierdurch bitte ich die Fachgenossen, ihnen bekannte Fehler in Sternkatalogen, die nicht in den astronomischen Zeitschriften, in den Annalen der.Sternwarten oder in Sternkatalogen veröffentlicht sind, mir baidgefälligst mitteilen zu wollen.

Berlin NW. 7, Dorotheenstr. 6, Bureau der Geschichte des Fixsternhimmels. I 904 März 2 I.

F. Ristenpart.

\section{Anzeige betr. Nr. 5 der Ergänzungshefte zu den Astr. Nachr.}

Nr. 5 der als Ergänzungshefte zu den Astronomischen Nachrichten herausgegebenen Astronomischen Abhandlungen, enthaltend W. Foerster, Beiträge zur Ausgleichung der fundamentalen Ortsbestimmungen am Himmel, ist erschienen und kann zum Preise von 3.50 Mark direkt von der Expedition in Kiel, Niemannsweg 103, oder durch die Buchhandlung W. Mauke Söhne in Hamburg bezogen werden.

Inhalt 2u Nr. 3938. E. Strömgren. Über die gegenseitigen Störungen zweier einander nahekommenden kleinen Planeten. I 7 . $-K$. Pokrozski, S. Scharbe. Bedeckung des Aldebaran I903 Okt. 10. 23. - F. Palisa. Vorschlag betr. die Anfertigung neuer Himmelskarten. 25. - E. Anding. Über die Schönfeldschen Hilfsgrößen im Problem der Bahnverbesserung. 29. - $M$. Wolf. Neuer Veränderlicher II.1904 Orionis. 29. - M. Wolf. Entdeckung zweier Veränderlicher. 31. - F. Palisa. Beobachtungen von Planeten auf der k. k. Sternwarte in Wien. 31. - M. Wolf. Nachträgliche Auffindung eines neuen Planeten 1902 NU. 31. - F. Ristenpart. Aufforderung betr. Fehler in Sternkatalogen. 31. - Anzeige betr. Nr. 5 der Ergänzungshefte zu den Astr. Nachr. $3 \mathbf{I}$.

Geschlossen rgo4 April 12. Herausgeber: H. Kreutz. Druek von C. Schaidt. Expedition: Kiel, Niemannsweg roz. 